\title{
Pre-knowledge of isolation as a prerequisite for the isolation-effect'
}

E. RAE HARCUM

THE COLLEGE OF WILLIAM AND MARY

\begin{abstract}
This study tests the hypothesis that prior knowledge of isolation is critical for an isolation-effect in perception. For some Os vertical lines were placed above and below the seventh of 10 elements in a tachistoscopic binary pattern. Other Os saw only patterns without such isolation, and a third group of Os saw both isolated and unisolated patterns in random sequence. An isolation-effect was found only for Os who viewed isolated patterns exclusively. Only these Os knew before exposure that the seventh element would be isolated. The isolation-effect in pattern perception, as in serial learning, was attributed to the selective distribution of attention among stimulus-elements.
\end{abstract}

\section{Problem}

To prove the existence of common mechanisms in serial learning and in the tachistoscopic perception of spatial patterns, Harcum \& Hartman (1961) attempted to find an isolation-effect in pattern perception. They did not find such an effect, however, apparently because their Os did not have sufficient foreknowledge about the isolation for each exposure. Results of Magne (1952) suggest that such knowledge is important for the isolation-effect in perception. In serial learning ordinarily the $\mathrm{S}$ can perceive the isolation on the first trial, and consequently he can know of it on subsequent trials. The isolation-effect apparently only occurs on the succeeding trials. Saltz \& Newman (1959) failed to find stronger intra-list associations for an isolate after only a single trial, although they did find evidence for increased attention to it on the second trial. Isolation, therefore, serves to direct effort selectively, rather than to make the isolated element intrinsically easier to learn (Jensen, 1962).

Harcum (1965b) predicted that an isolation-effect would be found in the tachistoscopic perception of binary patterns if $\mathrm{O}$ had prior knowledge of the isolation. He did find such an effect, but he did not establish with certainty the critical role of the foreknowledge of isolation, because other conditions were different from the Harcum \& Hartman (1961) experiment.

The present study tests the hypothesis that the isolation-effect is due to the selective direction of a perceptual process which scans the elements in sequence. Since this scan ordinarily proceeds from left to right across the visual field, a primacy effect favors elements at the left (Harcum, 1964). However, it is predicted that the primacy-effect will favor an isolated element, if it is known to be isolated before the exposure. When $O$ does not know when isolation will appear, there will be no difference between isolated and unisolated patterns. O may respond to all patterns either as isolated or as unisolated.

\section{Method}

The apparatus was much the same as that of Harcum (1965b). Stimuli were 10 circles in a horizontal line, with five circles to the right and left of fixation. Various five circles were blackened to form 20 binary patterns and their mirror images. Patterns were shown to each $O$ in the same random sequence, each pattern appearing four times. Exposure duration was $.1 \mathrm{sec}$. For six Os, called the Isolated (I) Group, all patterns contained vertical black lines above and below the seventh element from the left. Six differentOs, the Unisolated (U) Group, observed patterns without isolation lines. For a third group of six Os, the Mixed (M) Group, the isolating lines appeared on two of the four presentations of each pattern, randomly assigned throughout the sequence. The $O$ s in the $M$ and I Groups were instructed merely that some or all, respectively, of the patterns were accompanied by lines above and below the seventh circle from the left. In scoring the O's reproduction of the pattern of filled elements, errors of omission and commission were both counted.

\section{Results}

First tabulation of these data revealed an error in the selection of patterns. Some patterns contained groups of three adjacent open or filled elements, resulting in spuriously correct responses for some elements when errors in the localization of responses within the recording template occurred. This artifact was eliminated by analyzing only the data from targets having no more than two adjacent filled or unfilled circles. Although only 40 observations per $O$ remain, all of the present conclusions apply to the complete data also.

Proportions of errors at each circle-position for each group of Os are shown in Fig. 1. The ordinate is marked off in equal units, with sufficient identifying numbers to serve each set of curves. The curves labelled "Record" for the I and the U Groups refer to the corresponding 20 observations under isolated and unisolated conditions for the M Group, respectively. "Fill" curves refer to the remaining 20 observations. The two curves indicate the high reliability of results for each $O$, even under the two different conditions for the M Group. Thus, the loss of data from the eliminated patterns is not serious.

The U Group exhibits fewer errors at the left, as is usual for unisolated patterns (Harcum, 1964), but the 
I Group shows by comparison an isolation-effect around the seventh position. Since Element-position 4 is the mirror image of Position 7 , it can serve as a reference point for evaluating an effect of isolation. An isolation-effect is implied, therefore, by negative or low positive values for an $O$ when the totals of errors at Element-position 4 are subtracted from those at Position 7. The I Group is significantly different from the U Group on this measure by the Mann-Whitney test $(U=.66, p<.002)$. However, for the M Group, the isolated and unisolated conditions are obviously not different. The differences amounted to only two errors or less for five of the six Os, with three in the wrong direction. The M Group, with isolated and unisolated patterns combined, is significantly different from the I Group $(U=0, p=.001)$, but not from the $U$ Group $(\mathrm{U}=13.8, \mathrm{p}>.484)$.

The means of total errors per $O$ for the $M, I$, and U Groups are 139.8, 135.8, and 137.6, respectively. Since the smallest range of total errors among os within a group was 58, these means are obviously not significantly different. Within the M Group, the means of total errors per $O$ for isolated and unisolated patterns were 70.0 and 69.8 , respectively. This again is not significant, since the differences for individual Os ranged from 2 to 12 errors, equally distributed in plus and minus directions.

\section{Diseussion}

Since all predictions were confirmed, the present hypothesis is verified. Thus, the isolation-effect is produced by a selective mechanism which directs the attention to elements of a pattern in sequence. If the exposure-duration is not sufficiently long to permit the development of the selective perception, then the selectivity must be provided by pre-exposural information. Other studies (e.g., Camp \& Harcum, 1964) provide evidence against fixational errors as a cause of these differences in distributions of errors.

When the elements are arranged for each $O$ according to the rank order of their accuracy in reproduction, and then averaged, the functions are virtually identical for isolated and for unisolated targets, and they are approximately linear. This holds for the comparison of I and $U$ Groups, as well as for the comparison within the M Group. Consequently, as mentioned above, the totals of errors for isolated and unisolated patterns are not different. These are the same conclusions reported by Jensen (1962) for serial learning of colored forms by the anticipation method. They support Jensen's argument that isolation in serial learning merely changes the order in which individual items are learned.

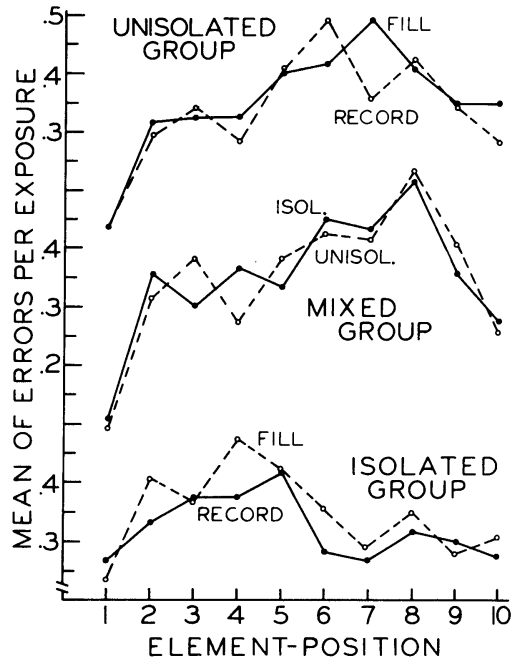

Fig. 1. Means of errors in reproducing elements at each position for each group of $O s$. The solid or dashed curves represent 20 corresponding observations

Thus, Harcum's (1965a) argument for the similarity of mechanisms in serial learning and pattern perception is also supported. The O's strategy in approaching either task, which can be influenced by certain stimuluscharacteristics, determines the shape of the curve of errors per element-position. In other words, isolation acts through its effect on the selective distribution of the $\mathrm{O}$ 's attention among the stimulus-elements.

\section{Referenees}

Camp, D. S., \& Harcum, E. R. Visual pattern perception with varied fixation locus and response recording. Percept. mot. Skills, $1964,18,283-296$.

Harcum, E. R. A curious parallel between serial learning and tachistoscopic perception. Virginia J. Sci., $1965^{\mathrm{a}}, 16,99-104$.

Harcum, E. R. An isolation effect in pattern perception similar to that in serial learning. Percept. Mot. Skills, $1965^{\mathrm{b}}, 20,1121$ 1130

Harcum. E. R. Reproduction of linear visual patterns tachistoscopically exposed in various orientations. The College of William and Mary: Williamsburg, Virginia, 1964

Harcum, E, R. \& Hartman, R. R. A serial-learning effect within the perceptual span Virginia J. Sci., 1961, 12, 203 (Abstract) Jensen, $\mathbf{A}, \mathbf{R}$. The von Restorff isolation effect with minimal response learning. J. exp. Psychol., 1962, 64, 123-125.

Magne, O. Perception and learning. Uppsala: Appelbergs Boktryckeri Ab, 1952

Saltz. E., \& Newman, S. E. The von Restorff isolation effect: test of the intralist association assumption. J. exp. Psychol., 1959, $58,445-451$

\section{Note}

1. This research was supported by a PHS research grant (HD 00207-06) from the Institute of Child Health and Human Development, Public Health Service. 